\title{
La internacionalización de Antioquia en el marco institucional ${ }^{*}$
}

\author{
The internationalization of Antioquia in the institutional framework
}

A internacionalizacao de Antioquia no marco institucional

Recibido el 14 de septiembre de 2015. Aceptado el 9 de octubre de 2015

\author{
Olga Lucía Zapata Cortés* \\ Colombia
}

\section{Resumen}

Objetivo: determinar la importancia de la internacionalización del departamento de Antioquia que se ha gestado desde la Gobernación para el logro de la promoción del desarrollo regional, bajo la perspectiva de globalización e internacionalización. Metodología: se hizo una revisión documental de los planes estratégicos y

, Para citar este artículo:

Zapata Cortés, Olga Lucía (diciembre, 2015).

La internacionalización de Antioquia en el marco institucional. Ánfora, 22(39), 45-

69. Universidad Autónoma de Manizales. ISSN 0121-6538. de los planes de desarrollo departamentales del período 2004-2015. Se revisaron los programas y proyectos que fueron definidos por la Gobernación de Antioquia con miras a lograr un cambio en el modelo de desarrollo en competitividad e internacionalización para la región. Resultados:duranteel período 2004-2015, los gobiernos departamentales establecieron programas y proyectos para la integración regional como el pilar necesario en la generación de oportunidades de internacionalización

\footnotetext{
* Este artículo es producto de investigación concluida: "Agenda pública para Antioquia 2012-2015. El caso de la alianza entre la Alcaldía de Medellín y la Gobernación de Antioquia", financiada por el Comité para el Desarrollo de la Investigación -CODI- de la Universidad de Antioquia, ejecutada entre el 5 de febrero de 2014 y el 5 de agosto de 2014. Acta 643 de 2012. Investigador principal: Olga Lucía Zapata Cortés.

** Magíster en Ciencia Política. Economista. Profesora de la Universidad de Antioquia. Grupo de Investigación Estudios en Gobierno y Corrupción. Correo electrónico: lucia.zapata@udea.edu.co. Grupo de Investigación Estudios en Gobierno y Corrupción.
} 
para el territorio. Se formalizó la Comisión Tripartita, instancia intergubernamental que viene consolidándose como la encargada de liderar la política regional de competitividad, productividad y la internacionalización de la región antioqueña. Conclusiones: si bien ha habido avances importantes, así como intentos de alianzas estratégicas entre Medellín y Antioquia, se presentan retos que van más allá de la capacidad institucional. Esto ha ocasionado un "derrame" de los beneficios de la internacionalización de Medellín hacia los municipios antioqueños más cercanos a la capital. En este sentido, el mayor esfuerzo económico y estratégico lo realiza Medellín, aprovechando la institucionalidad que ha sido creada para ese fin. En tanto, Antioquia aprovecha parte de esos beneficios a través de algunos de sus municipios más cercanos a la capital.

Palabras clave: Internacionalización, Paradiplomacia, Coordinación multinivel, Relaciones intergubernamentales.

\section{Abstract}

Objective: to determine the relevance of the internationalization of Antioquia introduced by the Departmental Government in order to achieve regional development promotion from the perspective of globalizationand internationalization. Methodology: a literature review of the strategic plans and the departmental development plans between 2004-2015 was carried out. Programs and projects that were defined by the Government of Antioquia, in order to achieve a change in the development model for competitiveness and internationalization intheregion, were reviewed. Results: between 2004 and 2015, departmental governments set up programs and projects for regional integration as a necessary foundation for generating internationalization opportunities in the territory. The Tripartite Commission, which is an intergovernmental body that is establishing itself as responsible for leading the regional policy of competitiveness, productivity and the internationalization of the Antioquia region, was formalized. Conclusions: although there has been significant progress, as well as attempts to establish strategic alliances between Medellin and Antioquia, challenges that go beyond the institutional capacity are presented. This has led to a "spillover" of the benefits of internationalization of Medellin to the municipalities belonging to Antioquia that are closest to the capital. In this regard, the greatest economic and strategic effort is made by Medellin, using the institutions that have been created for this purpose. Meanwhile, Antioquia takes advantage of those benefits through some of its municipalities that are closest to the capital.

Keywords: Internationalization, Paradiplomacy, Multilevel Coordination, Intergovernmental Relations. 


\section{Resumo}

Objetivo: determinar a importância da internacionalização do departamento de Antioquia que tem se gestado desde a Governação para o logro da promoção do desenvolvimento regional, sob a perspectiva de globalização e internacionalização. Metodologia: Fez se uma revisão documental dos planos estratégicos e dos planos de desenvolvimento departamentais do período 2004-2015. Revisaram-se os programas e projetos que foram definidos pela Governação de Antioquia a fim de lograr uma mudança no modelo de desenvolvimento na competitividade e internacionalização para a região. Resultados: durante o período 2004-2015, os governos departamentais estabeleceram programas e projetos para a integração regional como o pilar necessário na geração de oportunidades de internacionalização para o território. Formalizouse a Comissão Tripartite, organismo intergovernamental que vem consolidando-se como a encarregada de liderar a política regional de competitividade, produtividade e a internacionalização da região antioqueña. Conclusões: embora tenha havido progressos significativos, assim como tentos de alianças estratégicas entre Medellín e Antioquia, presentam se retos que vão além da capacidade institucional. Isto tem ocasionado um "derrame" dos benefícios da internacionalização de Medellín para os municípios antioqueños mais pertos da capital. Em este sentido, o maior esforço económico e estratégico é realizado por Medellín, aproveitando a institucionalidade que tem sido criada para esse fim. Enquanto, Antioquia aproveita parte desses benefícios a través de alguns de seus municípios mais pertos da capital.

Palavras chave: Internacionalização, Para-diplomacia, Coordenação multinível, Relações intergovernamentais. 


\section{Introducción}

El departamento de Antioquia ha formulado planes estratégicos con miras a un cambio en el modelo de desarrollo, en el cual la competitividad y la internacionalización de la región sean las grandes metas a lograr. Uno de los planes que se propuso estos objetivos fue el Plan Estratégico de Antioquia de 1998, el cual ya advertía como retos para el desarrollo de Antioquia, superar "el atraso tecnológico y la baja productividad, el aislamiento geográfico que castiga la competitividad, la precaria estabilidad interna en lo social y político, el mercado interno deprimido con macroeconomía inestable y la mentalidad y cultura poco inclinadas a la internacionalización" (Plan Estratégico de Antioquia, 2006, p. 22). Posteriormente, se implementaron otras iniciativas, como la Comisión Tripartita en 2004, para apoyar los procesos de internacionalización y mejoramiento de la productividad y competitiva en el departamento.

En este contexto, el proceso de internacionalización de Medellín es el que ha tenido mayor protagonismo y reconocimiento. Tal y como lo concluyen Molina et al (2012), la política de internacionalización de Medellín se instaló como un asunto permanente en la agenda gubernamental de la ciudad desde el 2004. Además, esta política es considerada como el factor fundamental para la transición del modelo de desarrollo económico basado en la industria hacia el de servicios. Se destaca entonces, que el éxito de esta política ha dependido en buena medida del fortalecimiento institucional local (la cual incluyó infraestructura, agencias especializadas, eventos, regeneración urbana y redes de trabajo) y del mercadeo territorial.

Ahora bien, tanto para el caso de Medellín como para el de Antioquia, ambos gobiernos han estado en constante interacción a través de sus planes de desarrollo, particularmente de las líneas y componentes que intentar articular la ciudad y la región mediante la integración regional. Ello se observa en el período 2004-2015, ya que los diferentes gobiernos departamentales han establecido programas y proyectos que buscan la integración regional, y más aún, la generación de "nuevas oportunidades de internacionalización para el territorio" (Cámara de Comercio, s.f., p. 1). Se trata de líneas de acción que tienen como objetivo materializar los principios de concurrencia, subsidiariedad y complementariedad que propone la Ley 152 de 1994, así como lograr el nuevo modelo de desarrollo propuesto por el Planea, en el que Medellín no siga concentrando la riqueza del departamento y que ésta se redistribuya en todas las subregiones. 
De ahí que los componentes y programas de integración regional resulten en opciones de trabajo conjunto (cuadro 1).

Particularmente, desde el gobierno de Antioquia la integración regional se ha fijado metas que van en concordancia con las mejoras en los niveles de competitividad, así como la inserción de Antioquia en los flujos económicos globales ${ }^{1}$. En este sentido, el gobierno de Aníbal Gaviria (2004-2007) se propuso como objetivo "Afirmar el territorio de Antioquia, articulándolo e integrándolo internamente y abriéndolo a las regiones vecinas, al país y al mundo”2, para lo cual promovería acciones en función de ampliar cobertura de servicios públicos, las telecomunicaciones y la infraestructura física y de transporte. Se trata pues de materializar el nuevo direccionamiento del departamento ${ }^{3}$, propuesto en el Planea, en cuanto a la integración regional y el desarrollo de Antioquia. En este período, se fortaleció la alianza entre la Gobernación, la Alcaldía y el Área Metropolitana mediante la formalización de la Comisión Tripartita para la internacionalización y posicionamiento estratégico de Antioquia, a través de la articulación de sus agendas territoriales. También se iniciaron acciones que buscaron potenciar a Medellín, entre ellas, los clúster, las sedes universitarias subregionales, el fomento a los emprendimientos.

La Comisión Tripartita se ha consolidado como la instancia intergubernamental encargada de liderar la política regional de competitividad y productividad del departamento, así como la internacionalización de la región antioqueña. Para ello, propone lineamientos y políticas que son implementadas en el territorio, algunas de las cuales con el consenso de los sectores gubernamentales, empresariales y privados, aprovechando la participación activa que en esta instancia encuentran los empresarios antioqueños y el sector privado representados por organizaciones como la Cámara de Comercio y Proantioquia.

En el gobierno de Luis Alfredo Ramos (2008-2011), la internacionalización de la región se trazó desde el objetivo de lograr una "Adecuada Integración del Departamento de Antioquia al Contexto Mundial”, en el que iba a tener una gran importancia los programas y acciones a favor de la productividad y la

\footnotetext{
1. Para más información acerca de estos objetivos, consúltese: http://antioquia.gov.co/index.php/prensa/ historico/63-plan-de-desarrollo/linea-estrategica-3/2519-internacionalizacion.

2. Plan de Desarrollo 2004-2007, "ANTIOQUIA NUEVA, un hogar para la vida.

3. Como parte de esta propuesta, se planteó la revisión de las vocaciones económicas de las subregiones y los municipios, de manera que adquirieran fortalezas para incrementar su productividad y competitividad.
} 
competitividad, tales como: la Agenda Interna (para definir e impulsar sectores productivos), los Sistemas Productivos Locales -SPL- para la exportación y para afrontar la competencia externa (para generar redes empresariales), la formación de capital humano (formación de empresarios y emprendedores y bilingüismo), la articulación público-privada (la Comisión Tripartita, el Acuerdo de voluntades y la Comisión Regional de Competitividad) y el Plan Integral de Marketing Territorial (incluye ferias internacionales). Además, también sobresalieron programas como: Implementación de redes de actores subregionales que gestionen la productividad y competitividad, Impulso a la infraestructura para la productividad y competitividad en las subregiones, Cadenas productivas, Mejoramiento de la competitividad de la minería para la internacionalización, y Vías para la competitividad en Antioquia.

En esta primera parte, se presenta una breve descripción de los elementos teóricos, centrados en los enfoques de los actores gubernamentales no centrales y las relaciones intergubernamentales. El primer enfoque se centra en los desarrollos teóricos que ha tenido la paradiplomacia, la cual se ha constituido en un enfoque explicativo de las relaciones internacionales que emprenden los gobiernos locales y regionales. Es definida por Cornago (2004)como "el involucramiento de los gobiernos subestatales en las relaciones internacionales, por medio del establecimiento de contactos formales e informales, permanentes o provisorios (ad hoc), con entidades extranjeras, públicas o privadas, con el objeto de promover resultados socioeconómicos o políticos, tanto como cualquier otra dimensión externa de su propia competencia constitucional" (Romero, 2005, p.56). En ese sentido, se entienden como acciones diplomáticas paralelas que ejercen unidades de gobierno diferentes del nivel nacional, también denominados como actores gubernamentales no centrales, y "que está directamente relacionada con el establecimiento de relaciones potencialmente benéficas desde el punto de vista del desarrollo de las entidades locales involucradas" (Molina et al, 2010, p. 35). $\mathrm{Al}$ respecto, Morales (2013) plantea que la motivación de la paradiplomacia de los gobiernos no centrales está orientada al desarrollo local, por lo que incluyen estrategias como las marcas y el marketing de ciudades para promocionar el turismo, las inversiones o intercambios culturales, entre otros.

Este incremento de las acciones internacionales desplegadas por los gobiernos locales y regionales fue de gran interés académico para los estudiosos de la política comparada y las relaciones internacionales como referentes teóricos para explicar la participación de los gobiernos no centrales (Farías, 2014). Y aunque en la literatura se pueden encontrar términos como microdiplomacia, diplomacia multinivel, postdiplomacia y mesogobiernos, por ejemplo, el término 
paradiplomacia emergió con fuerza en 1986, tal como lo explican Molina et al (2010), cuando Duchacek fracasó al proponer el término microdiplomacia para explicar el fenómeno de las relaciones por fuera de los Estados nación de las unidades subestatales. Por otro lado, el enfoque de la paradiplomacia enfrenta dos paradigmas de las relaciones internacionales: "uno relacionado con la interdependencia compleja, que contempla el pluralismo de actores, es decir el de la sociedad mundial, y el otro, Estado-céntrico, llamado también tradicional, basado en el realismo político" (Rodríguez, 2004, p. 6).

El impulso de la paradiplomacia fue favorecido por factores contex tuales como "la crisis del Estado y la globalización, el internacionalismo y la integración regional al igual que el nacionalismo, planteando dos elementos adicionales que alientan su desarrollo: los asuntos transfronterizos y el hermanamiento de ciudades" (Farías, 2014, p. 159). Agrega Ugalde (2006) que la introducción de Actores Gubernamentales No Centrales (AGNC) en las relaciones internacionales florece debido al debilitamiento de la centralidad del Estado, lo que genera nuevas realidades no sólo en las relaciones internacionales, sino también en las relaciones intergubernamentales favorecidas, porque en la actualidad se conjugan los procesos de integración regional y descentralización, más conocidos como "glocalización", tal como lo argumenta Esposto (2006). Así, los procesos de glocalización habrían permitido, desde la perspectiva de Soja (2008), que los gobiernos locales tuvieran "una visión global adaptada a las condiciones locales o a la localización de la globalidad" (p. 289) en el marco de las nuevas configuraciones de las geografías del poder. En otras palabras, se trata de diversas relaciones posibles entre lo global y lo local, con especial interés en las de tipo económico de carácter regional.

Sin embargo, "no debe pensarse el ascenso de la paradiplomacia como el declive de la diplomacia estatal, sino que se trata de un conjunto de prácticas de interrelación transnacionales que se superponen parcialmente a aquella y complejizan el escenario político mundial” (Ferrero, 2006, p. 7).La participación de estos actores (AGNC) se caracteriza por tener objetivos asociados a "razones políticas (como una futura independencia), culturales (promoción de su idioma y cultura) y económicas (exportación de sus productos, atracción de inversiones y promoción turística). Asimismo, agrega que las motivaciones fronterizas (gestión de problemas comunes) y migratorias" (Farías, 2014, p. 160).

Las diferentes motivaciones se pueden diferenciar según la clasificación propuesta por Ugalde (2015) en: a) las de origen externo; que comprenderían razones asociadas a los efectos de la globalización, grados de interdependencia y 
procesos de integración supraestatal que se generan al interior de las estructuras estatales: Políticas de los gobiernos no centrales ante la globalización, Medidas para afrontar un entorno económico y comercial competitivo, Actividades de otros actores internacionales y Participación en estrategias de integración regional. b) las de origen interno, producidas por las transformaciones del Estado-nación. Éstas, a su vez, se dividen en i) las relacionadas al conjunto del Estado: Procesos de descentralización, Conflictos entre el gobierno central y los gobiernos locales y departamentales, y las Dificultades de los gobiernos subnacionales para incidir en la posición internacional del Estado; ii) las producidas en cada nivel de gobierno: Características políticas, culturales, económicas y sociales distintivas, Competencias asumidas y su proyección exterior, Posiciones regionalistas o autonomistas independentistas y el "yo también", es decir, la imitación de estrategias de internacionalización que ya han sido desplegadas por otros gobiernos.

Las diferentes estrategias o actividades que los actores gubernamentales no centrales pueden implementar en el marco de la paradiplomacia son: 1) Viajes al extranjero, visitas recibidas y actividades promocionales y de fomento exterior. 2) Delegaciones en el exterior. 3) Firma de acuerdos y convenios exteriores. 4) Cooperación interregional multilateral y creación de asociaciones interregionales. 5) Cooperación transfronteriza. 6) Participación de los GNC en las organizaciones de integración supraestatales. 7) Creación de asociaciones y redes mundiales de GNC. 8) Presencia de los GNC en las Organizaciones Internacionales intergubernamentales, y 9) Cooperación al desarrollo y ayuda humanitaria (Ugalde, 2006, pp. 124-126).

Tal como las describe Ugalde (2006), estas actividades van desde las más básicas, pasando por las de tipo alianzas sectoriales y compromisos de largo plazo con agencias de cooperación y fondos financieros. Tienen como propósitos establecer contactos e intercambios que puedan asegurar una eficaz promoción de ciudades y regiones, enmarcadas en estrategias de internacionalización. Además, crear y participar en asociaciones, así como firmar acuerdos y convenios para proyectos de cooperación de tal manera que se intervengan problemáticas sociales comunes. Para el caso de las ciudades, Borja y Castells (1997) enuncian como las principales estrategias que las ciudades deberían implementar para internacionalizarse las siguientes:

i) Tener una participación activa en eventos internacionales, ii) participar en asociaciones de ciudades, iii) constituir redes de ciudades, iv) participar en procesos de integración regional, y v) tener presencia directa en el sistema de cooperación 
internacional" (Vieira, 2011, p. 1). Esto converge con el siguiente supuesto planteado por Milani y Ribeiro (2010):"Las ciudades, gracias a sus redes y proyectos de cooperación transnacionales, constituyen la expresión de un actor político nuevo tras cambiar la escala en que operan y emanciparse parcialmente del monopolio ejercido por el Estado-nación en la administración pública transfronteriza (Borja y Castells, 1997, p. 25).

También, desde la teoría de las Relaciones Intergubernamentales (RIG), se pueden destacar elementos de relacionamiento multinivel que permiten explicar el proceso de internacionalización de Antioquia en el marco de la organización estatal colombiana, a través del principio de separación de los niveles de gobierno, en el que cada instancia conserva facultades para la toma de decisiones políticas y es considerada como un actor del sistema político. Desde esta perspectiva, se resalta la autonomía de los municipios, al igual que la necesidad de mecanismos de coordinación y articulación de los diferentes niveles de gobierno. En este sentido, las RIG son definidas como "el conjunto de nexos e intercambios emergentes de la interrelación entre los distintos niveles de gobierno, instancias en las que se determinan políticas y estrategias generales de desarrollo en circunscripciones territoriales determinadas" (Arandia, 2002, p. 10). Así que esta teoría facilita la comprensión de las formas de relacionamiento entre el gobierno departamental y municipal en Antioquia, orientadas a la descentralización y a la delegación de funciones y competencias de los entes territoriales, centradas en una perspectiva oficialista e institucional.

Las RIG pueden analizarse según Wright (1997) desde tres modelos principales: de autoridad coordinada (autonomía), de autoridad dominante o inclusiva (jerárquica) y de autoridad igual o superpuesta (negociación). Pero no todas las relaciones entre niveles de gobierno son formales y jerárquicas, ya que el concepto "no contiene distinciones jerárquicas de superioridad o inferioridad. $\mathrm{Y}$ aunque no excluye la existencia de diferencias de poder, tampoco implica que el nivel nacional sea, presuntamente, el superior" (1997, p.101). Otra clasificación es propuesta por Arandia (2002): i) entre instancias de gobierno de un mismo nivel $\mathrm{u}$ horizontales $\mathrm{y}$, ii) entre instancias de gobierno de distintos niveles o verticales. Las relaciones del segundo tipo, son aquellas que se dan entre el gobierno central, los gobiernos intermedios (departamentos) y los gobiernos locales (municipios). Aunque Arandia enuncia que la noción de verticalidad está dada por las funciones y competencias que cada nivel ejerce, para el caso colombiano, la verticalidad o jerarquía están dadas por factores como el territorial, el normativo, el administrativo, el financiero, el político, entre otros. 
En cuanto a los canales de relacionamiento, sobresalen el canal formal y el informal. Los canales formales se manifiestan mediante procedimientos de asignación, coordinación, delegación y decisiones institucionalizadas sobre recursos, competencias, políticas sectoriales, reparto de poder, entre otros. Por su parte, los canales no formales "se materializan en la coordinación informal entre los representantes y responsables de los distintos niveles de gobierno a través de sus partidos políticos" (Arandia, 2002, p. 12), por lo que se crean redes intergubernamentales entre las comunidades de profesionales responsables de las políticas públicas descentralizadas. Jordana (2002a y 2002b) plantea que las reglas electorales y los partidos políticos harían parte de los arreglos formales e institucionales; mientras que los arreglos denominados de carácter "informal" conformados por profesionales, representantes políticos y otros actores del sistema, se mantendrían dentro de la dimensión informal.

La teoría gubernamentalista suministra elementos para hacer la lectura de las relaciones entre diferentes niveles de gobierno, conservando la jerarquía y la centralización de ciertas funciones. Así, "las relaciones intergubernamentales sirven para articular un ejercicio concertado de las competencias entre distintos centros de poder que permite resolver problemas" (García, Montilla y Arbós, 2006, p. 9). Para ello, las relaciones entre niveles de gobierno pueden fomentarse desde mecanismos formales y jerárquicos, que es lo usual; pero también pueden aprovechar mecanismos informales de negociación y cooperación no atravesados por la subordinación jerárquica.

Teniendo en cuenta estos elementos teóricos, se pretende responder las siguientes preguntas orientadoras: ¿cuál ha sido la apuesta institucional que ha realizado la Gobernación de Antioquia para lograr la internacionalización del departamento?, ¿Cuál ha sido el papel del departamento en la internacionalización de Medellín y cómo se ha beneficiado? ¿Cuál es la conexión entre el objetivo de internacionalización del departamento y la integración regional para mejorar la competitividad? Responder estas cuestiones permite evidenciar los resultados y retos alrededor de metas como la internacionalización de ciudades y regiones, así como los avances y problemas de la coordinación multinivel y el establecimiento de agendas articuladas que busquen alcanzar mayores niveles de desarrollo regional y local. 


\section{Metodología}

La presente investigación se desarrolló desde un enfoque cualitativo de tipo descriptivo, cuya modalidad investigativa se centró en el análisis documental, el cual se desarrolló en cinco etapas, tal como lo sugiere Sandoval (1996). En la primera, se realizó el rastreo e inventario de los documentos existentes y disponibles; en la segunda, se clasificaron los documentos identificados; en la tercera, se seleccionaron los documentos pertinentes para los propósitos de la investigación; en la cuarta, se realizó una lectura en profundidad del contenido de los documentos seleccionados, para extraer elementos de análisis y consignarlos en fichas bibliográficas. Finalmente, en el quinto paso, se realizó una lectura cruzada y comparativa de los documentos en cuestión, ya no sobre la totalidad del contenido de cada uno, sino sobre los hallazgos previamente realizados, de modo que fuera posible construir una síntesis comprensiva total del objeto analizado.

Las fuentes de información priorizadas fueron los planes de desarrollo de Antioquia, y sus respectivos informes de rendición de cuentas, correspondientes al período 2004-2014, el Plan Estratégico de Antioquia (Planea), los planes estratégicos subregionales, documentos institucionales relacionados con la internacionalización de Medellín y Antioquia, documentos públicos como ordenanzas, acuerdos y demás de tipo oficial sobre agencias gubernamentales que tienen competencias para la internacionalización de la región, otros documentos con información sobre el objeto de la investigación. Documentación sobre programas y proyectos definidos por la Gobernación de Antioquia con miras a un cambio en el modelo de desarrollo en el cual, la competitividad y la internacionalización de la región. La información se procesó en el software de N-Vivo.

Se hizo una revisión exhaustiva de los programas y proyectos formulados en los planes de desarrollo de Antioquia, centrando la mirada no sólo en los objetivos y metas, sino en la conexión que estos tenían con los planes estratégicos departamentales y subregionales, así como con los planes departamentales anteriores, para establecer continuidades. 


\section{Resultados}

Se presentan los resultados iniciando con una relación de apuestas institucionales delargo plazo, como el caso del Planea, que han propugnado por un cambio en el modelo económico regional y con ello, implementar estrategias de internacionalización de la región. Posteriormente se presenta el tratamiento que cada uno de los tres gobiernos que se revisan le ha dado a la estrategia de internacionalización y su conexión con la estrategia de integración regional, así como la política de internacionalización de Medellín. Finalmente, se expone el caso de la Alianza Medellín-Antioquia, que tenía la pretensión de incidir significativamente en la manera de direccionar la estrategia de internacionalización de la región en el actual gobierno de Sergio Fajardo, lo que le ha generado nuevos retos a este objetivo.

El departamento de Antioquia le ha apostado a la internacionalización de la región de manera sistemática desde la década de 1998, producto de los cambios económicos y globales que han presionado por la liberación y globalización de mercados y flujos de capital (Sassen, 2010). Uno de esos primeros esfuerzos por proponer una agenda para la internacionalización de Antioquia de largo plazo fue el Plan Estratégico de Antioquia, formalizado en 1998 mediante la Ordenanza $\mathrm{N}^{\circ}$ 12. En este documento se sustenta dicha agenda desde la necesidad de aprovechar las ventajas relacionadas con la vocación industrial del departamento, así como su capacidad por penetrar mercados internacionales en sectores como el café, las flores, alimentos y confecciones. Sin embargo, la mayor motivación se fundamentaba en la necesidad de atacar desventajas como: "el atraso tecnológico y la baja productividad, el aislamiento geográfico que castiga la competitividad, la precaria estabilidad interna en lo social y político, el mercado interno deprimido con macroeconomía inestable y la mentalidad y cultura poco inclinadas a la internacionalización" (Planea, 2006b, p. 22). Pero, en ese Plan no sólo se propendía por la internacionalización de la región, sino además por su ciudad capital, Medellín, en una relación de beneficios mutuos; aunque con el tiempo, la internacionalización de la ciudad fue de mayor visibilidad que la de la región.

La internacionalización se promovería desde dos de las cuatro líneas estratégicas definas por el Planea, esto es: Integrar y articular territorialmente a Antioquia y Revitalización de la economía antioqueña. La primera línea, propendería por la articulación de todo el territorio antioqueño, con el propósito de mejorar su competitividad y participar de los flujos económicos nacionales e internacionales; por su parte, desde la segunda línea, se fortalecería el mercado regional y se penetrarían mercados externos (Planea, 2006a). Ambas líneas han sido incorporados en los planes de desarrollo de Antioquia a partir del año 
2000, con programas y proyectos cada vez más ambiciosos en cuanto a metas de articulación territorial e internacionalización, sustentadas principalmente en equipamiento e infraestructura física, así como estrategias para mejorar la competitividad y productividad.

Cuadro 1. Tabla comparativa de planes de desarrollo Antioquia 2004-2015: Integración Regional e internacionalización

\begin{tabular}{|c|c|c|c|}
\hline $\begin{array}{l}\text { Compo- } \\
\text { nente }\end{array}$ & $\begin{array}{l}\text { "Antioquia nueva, un } \\
\text { hogar para la vida } \\
\text { 2004-2007" } \\
\text { Plan de Desarrollo } \\
\text { 2004-2007 }\end{array}$ & $\begin{array}{c}\text { "Manos a la Obra" } \\
\text { Plan de Desarrollo } \\
\text { 2008-2011 }\end{array}$ & $\begin{array}{c}\text { "Antioquia } \\
\text { La más Educada" } \\
\text { Plan de Desarrollo } \\
\text { 2012-2015 }\end{array}$ \\
\hline $\begin{array}{l}\text { Integración } \\
\text { Regional }\end{array}$ & $\begin{array}{c}\text { Línea 1. Integrar y articu- } \\
\text { lar territorial a Antioquia } \\
\text { OBJETIVO ESTRATÉ- } \\
\text { GICO } \\
\text { Afirmar el territorio de } \\
\text { Antioquia, articulándolo } \\
\text { e integrándolo interna- } \\
\text { mente y abriéndolo a las } \\
\text { regiones vecinas, al país y } \\
\text { al mundo. } \\
\text { OBJETIVO ESPECíFICO } \\
1.2 \\
\text { Inducir un proceso de } \\
\text { ocupación más adecuado } \\
\text { en el territorio antio- } \\
\text { queño. } \\
\text { OPERACIÓN Y ACCIO- } \\
\text { NES: } \\
\text { •Diseño, concertación y } \\
\text { adopción del plan depar- } \\
\text { tamental de ordenación } \\
\text { territorial. }\end{array}$ & $\begin{array}{c}\text { LÍNEA ESTRATÉGICA 4. } \\
\text { DESARROLLO TERRITO- } \\
\text { RIAL } \\
\text { OBJETIVO GENERAL: } \\
\text { Contribuir a un desarrollo } \\
\text { territorial equilibrado, } \\
\text { incluyente, sustentable am- } \\
\text { bientalmente, y articulado } \\
\text { interna y externamente. } \\
\text { Componente: } \\
\text { ORDENAMIENTO } \\
\text { REGIONAL. } \\
\text { OBJETIVO ESPECIFICO: } \\
\text { Avanzar en la construcción } \\
\text { de un modelo de desarrollo } \\
\text { territorial incluyente que } \\
\text { reconozca la heteroge- } \\
\text { neidad y diversidad de las } \\
\text { subregiones mediante la } \\
\text { promoción de las dinámicas } \\
\text { de desarrollo local, subregio- } \\
\text { nal y departamental. } \\
\text { local. } \\
\text { Programa 2: Definición y } \\
\text { desarrollo de herramientas } \\
\text { de planificación, gestión y } \\
\text { financiación de proyectos } \\
\text { necesarios para el desarrollo } \\
\text { y dotación del territorio. }\end{array}$ & $\begin{array}{c}\text { Línea estratégica 7. } \\
\text { Antioquia Sin Fronteras } \\
\text { OBJETIVO: Promover el } \\
\text { desarrollo regional a partir } \\
\text { de procesos de integración, } \\
\text { al interior del departamento } \\
\text { entre municipios y } \\
\text { subregiones, con los } \\
\text { departamentos y regiones } \\
\text { limítrofes con las que } \\
\text { Antioquia comparte } \\
\text { intereses,y con territorios } \\
\text { de países vecinos, } \\
\text { especialmente de Panamáy } \\
\text { de la Cuenca del Caribe, } \\
\text { en una perspectiva } \\
\text { de globalizacióne } \\
\text { internacionalización. } \\
\text { Componente: Acuerdos } \\
\text { para el desarrollo territorial } \\
\text { y sectorial. } \\
\text { PROGRAMA: ALIANZAS } \\
\text { CONDEPARTAMENTOS } \\
\text { VECINOS SOBRE } \\
\text { TERRITORIOS } \\
\text { COMPARTIDOS. } \\
\text { Proyecto: Esquemas } \\
\text { asociativos territoriales. } \\
\text { Proyecto: Fortalecimiento } \\
\text { de capacidades de gestión } \\
\text { y de planificación para el } \\
\text { desarrollo de "Antioquia sin } \\
\text { fronteras". }\end{array}$ \\
\hline
\end{tabular}




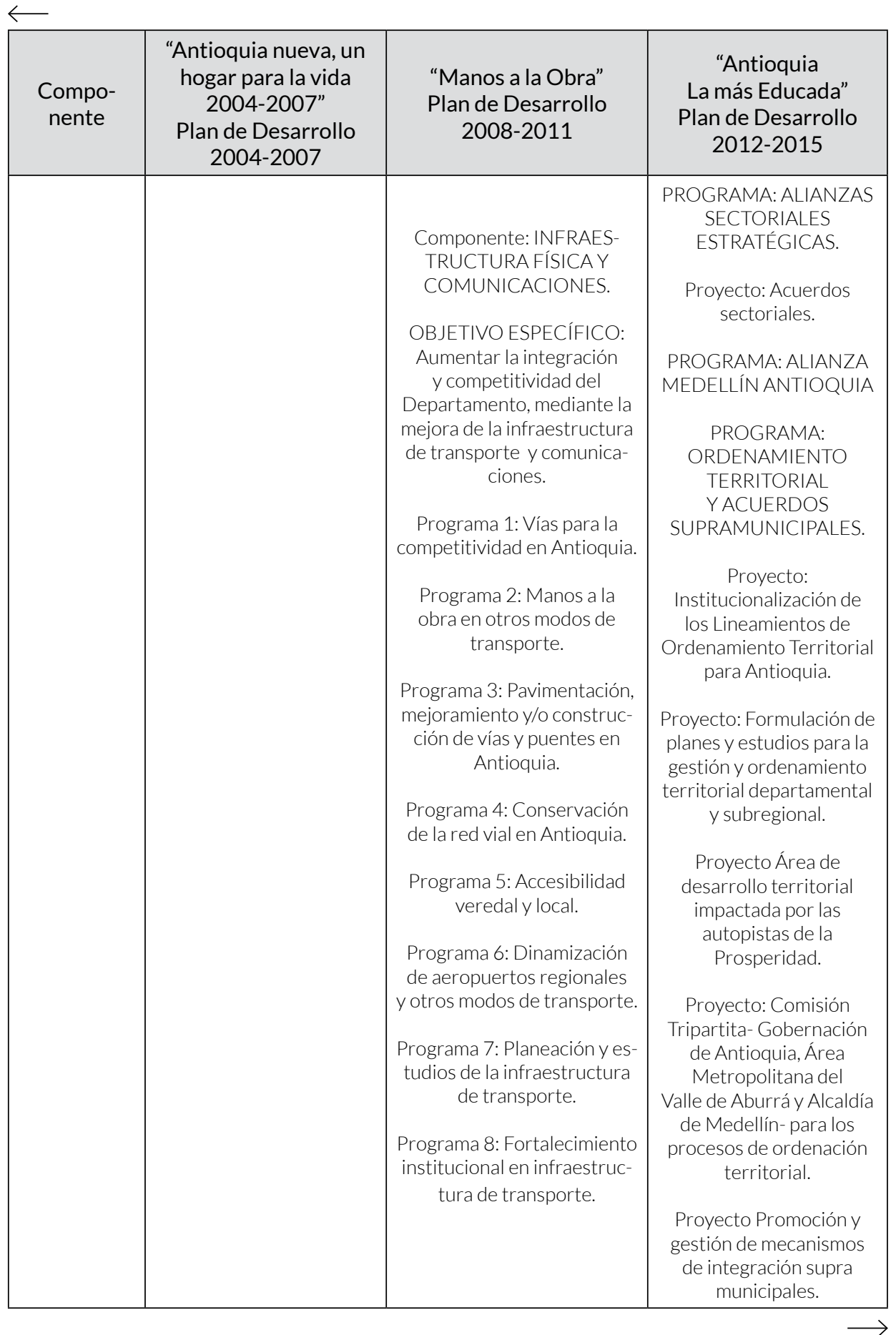




\begin{tabular}{|c|c|c|c|}
\hline $\begin{array}{c}\text { Compo- } \\
\text { nente }\end{array}$ & $\begin{array}{c}\text { "Antioquia nueva, un } \\
\text { hogar para la vida } \\
\text { 2004-2007" } \\
\text { Plan de Desarrollo } \\
\text { 2004-2007 }\end{array}$ & $\begin{array}{c}\text { "Manos a la Obra" } \\
\text { Plan de Desarrollo } \\
2008-2011\end{array}$ & $\begin{array}{c}\text { "Antioquia } \\
\text { La más Educada" } \\
\text { Plan de Desarrollo } \\
2012-2015\end{array}$ \\
\hline $\begin{array}{l}\text { Internacio- } \\
\text { nalización }\end{array}$ & 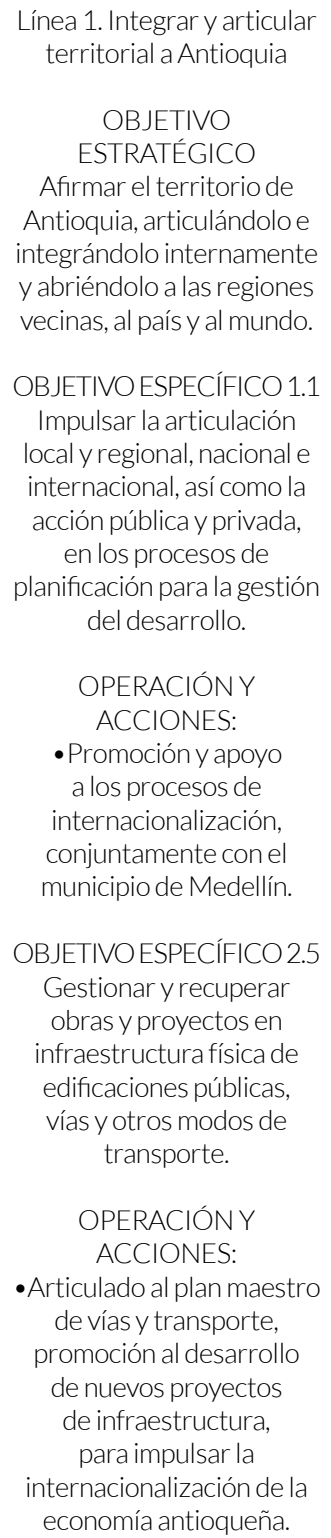 & 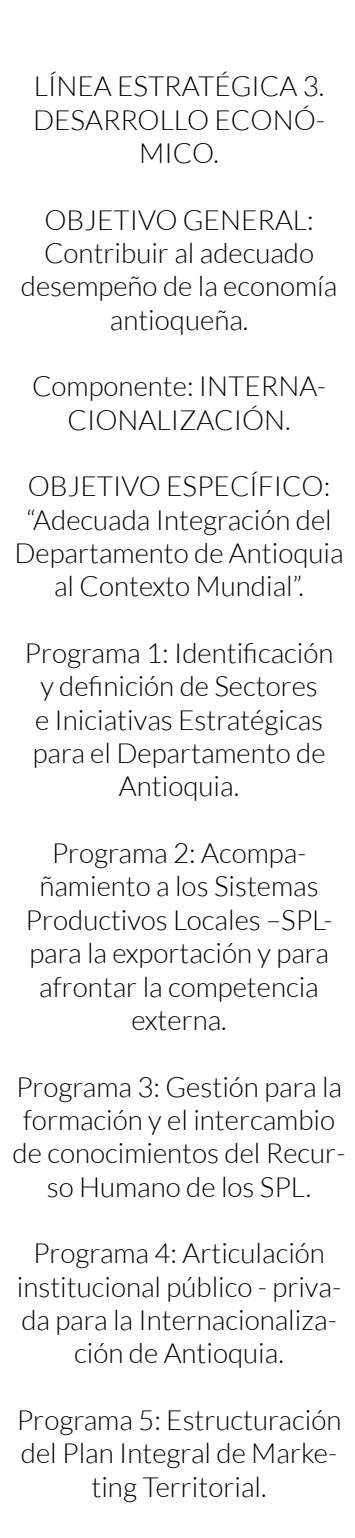 & $\begin{array}{l}\text { Línea estratégica 7. Antioquia } \\
\text { Sin Fronteras } \\
\text { OBJETIVO: Promover el } \\
\text { desarrollo regional a partir } \\
\text { de procesos de integración, al } \\
\text { interior del departamento } \\
\text { entre municipios y } \\
\text { subregiones, con los } \\
\text { departamentos y regiones } \\
\text { limítrofes con las que } \\
\text { Antioquia comparte } \\
\text { intereses, y con territorios de } \\
\text { países vecinos, especialmente } \\
\text { de Panamá y } \\
\text { de la Cuenca del Caribe, } \\
\text { en una perspectiva } \\
\text { de globalizacióne } \\
\text { internacionalización. } \\
\text { Componente: Antioquia } \\
\text { Internacional. } \\
\text { PROGRAMA: } \\
\text { INTERNACIONALIZACIÓN. } \\
\text { Proyecto: } \\
\text { el comercio y la cooperación } \\
\text { internacional. }\end{array}$ \\
\hline
\end{tabular}

Fuente: elaboración propia, a partir de los planes de desarrollo de Antioquia para el período 2004-2015. 
Por ejemplo, en el gobierno de Aníbal Gaviria, 2004-2007, se implementaron programas orientados a la integración regional como apoyo a la internacionalización de Medellín con acciones orientadas a la infraestructura de vías y transporte. Luego, en el gobierno de Luis Alfredo Ramos, 20082011, se incrementaron los programas dirigidos a la integración regional y la internacionalización de la región y a la ciudad de Medellín con acciones no sólo referidas a la infraestructura sino a otros asuntos como los sistemas productivos locales y exportaciones. Sin embargo, bajo este gobierno se propone la Estructuración del Plan Integral de Marketing Territorial con el cual se avanzaría en la definición de estrategias para la internacionalización regional.

Ahora bien, el actual Gobernador de Antioquia, Sergio Fajardo 4 , mediante la Linea 7. Antioquia sin fronteras de su Plan de Desarrollo 2012-2015: Antioquia la más educada, se ha propuesto:

Promover el desarrollo regional a partir de procesos de integración, al interior del departamento entre municipios y subregiones, con los departamentos y regiones limítrofes con las que Antioquia comparte intereses, y con territorios de países vecinos, especialmente de Panamá y de la Cuenca del Caribe, en una perspectiva de globalización e internacionalización (DAP, 2012, p. 263).

Uno de los programas que componen la línea se denomina Internacionalización (cuadro 1), que busca poner a Antioquia en el mapa del mundo con el apoyo de la Agencia de Cooperación e Inversión (ACI) para "la atracción de inversión, la participación en ferias y las misiones internacionales" (DAP, 2012, p. 266). Este objetivo se mediría a través del indicador: Empresas instaladas con base y operación en Antioquia, cuya meta es de nueve empresas para el cuatrienio. El programa se compone del proyecto Promoción de la inversión, el comercio y la cooperación internacional (cuadro 1), que busca atraer inversión mediante la cooperación sur-sur y mejorar las relaciones internacionales "para que Antioquia empiece a ser reconocida como una región con oportunidades en comercio y servicios, y en constante transformación" (DAP, 2012, p. 267). El total de la inversión del programa Internacionalización es de \$1.973 millones para el cuatrienio.

Según los informes de rendición de cuentas del gobierno Fajardo (20122015), algunos de los avances que se han logrado en la internacionalización de

\footnotetext{
4. Ex alcalde de Medellín en el período 2004-2007. Su método se basa en ejecutar en el departamento de Antioquia, las políticas y programas que tuvieron buenos resultados en Medellín, cuando él fue alcalde. De ahí, que aspire a una política de internacionalización como la formulada en la ciudad.
} 
Antioquia se traducen en acciones que ha adelantado la Gobernación en materia de relaciones internacionales con la European Commission, el Banco Mundial, el Banco Interamericano de Desarrollo, el Programa de las Naciones Unidas para el Desarrollo, la USAID (United States Agency Internacional Development), la ACNUR (Agencia de la ONU para los Refugiados), la International Youth Foundation, la Japan International Cooperation Agency, la Cepal, el Fondo Multilateral de Inversiones, el Ajuntament de Barcelona, entre otras (Informe de rendición de cuentas, 2014, p. 27).

Sin embargo, formular una política pública de internacionalización para Antioquia tal como fue planteada para Medellín presenta retos que van más allá de la creación de una institucionalidad e infraestructura propia. En el caso de Medellín, los avances en su internacionalización se lograron debido a la creación de la Agencia de Cooperación Internacional, el Medellín Convention Bureau y el Centro Internacional de Convenciones, los cuales se acompañaron de programas dirigidos a la creación de redes de personas e instituciones que promocionaran la ciudad en el exterior, bilingüismo, conectividad y TIC`s, así como fomento de eventos internacionales. Estrategias que, según Ugalde (2015), están acordes con las que suelen adelantar los AGNC en sus procesos de internacionalización.

Particularmente, la realización de eventos internacionales ha sido una de las estrategias más exitosas en el proceso de internacionalización de la ciudad. Y es que tan solo para el año 2014 se "programaron 90 eventos de gran magnitud en Medellín con un presupuesto de 11.000 millones de pesos" (Medellín Convention\&Visitors Bureau, 2014). Algunos de los eventos internacionales más reconocidos que se han realizado en Medellín en años recientes, además de los tradicionales Colombiamoda e Inexmoda que se realizan cada $a n ̃ 0^{5}$, han sido:

- La VII Conferencia Latinoamericana y Caribeña de Ciencias Sociales del CLACSO en 2015.

- La Asamblea de la Organización Mundial del Turismo en 2015, en la que se espera recibir a más de 3.000 visitantes.

- El Séptimo Foro Mundial Urbano en 2014, con más de 25.000 asistentes, entre los cuales estuvieron 50 ministros de Estado y 500 alcaldes del mundo.

5. También vale la pena mencionar eventos anuales como la Feria de las flores y el Festival Internacional de Poesía de Medellín. 
- El Segundo Foro Internacional por el Desarrollo de los Mercados de la Base de la Pirámide en América Latina del Banco Interamericano de Desarrollo (BID) en 2013.

- Los IX Juegos Suramericanos en 2010, que también se desarrollaron en subsedes por fuera de la ciudad capital, dando paso a municipios como Bello,Copacabana, Envigado, Guarne, Guatapé, Itagüí, Rionegro y Sabaneta para que participaran de los beneficios de este evento.

Otros eventos de gran importancia, según Medellín Convention\&Visitors Bureau, fueron: el concierto de Madona en 2013, "que atrajo a 32.000 visitantes y llegó que la tasa de ocupación promedio encima del $85 \%$, e incluso cercaba al $100 \%$ en algunas zonas de la ciudad, según cifras de Cotelco"; 17congresos, convenciones y conferencias mundiales, que representan un $62 \%$ de la meta de captación del año. De ahí que para el 2013, según el informe de la Asociación Internacional de Congresos y Conferencias (ICCA), Medellín haya subido "35 posiciones en el ranking mundial de países y ciudades que se destacan por su alta capacidad para la realización de eventos asociativos, pasando de la posición 120 a la 85" (El Colombiano, 2013, mayo 8).

A pesar de los programas y metas en relación con la integración regional en Antioquia, homologar la realización de eventos internacionales por parte de otros municipios antioqueños no es tarea fácil. Ello requeriría de inversiones sistemáticas en infraestructura hotelera y turística, así como el mejoramiento en infraestructura vial y aeroportuaria para las conexiones internas y externas, a pesar de los avances que se han reportado para el período 2004-2011: Por ejemplo, en 2005, el total de la red de carreteras era de 6.399 kilómetros, de los cuales 892 estaban pavimentadas; mientras que en 2011 el total ascendió a 19.962 kilómetros, de los cuales 1.436 estaban pavimentadas. En cuanto al transporte aéreo, el Anuario Estadístico de Antioquia (2011a), muestra que este "presentó a lo largo del periodo 2005-2011 una tendencia creciente, influenciada por el aumento en el movimiento de pasajeros salidos en Antioquia, pues para 2009 se incrementó en un 8,5\% con respecto al año anterior". También en el sector servicios, el subsector de hoteles, restaurantes, bares y similares:

muestra crecimientos positivos en todos los años entre 2005 y 201 1, siendo 2009 el año en que presentó el menor crecimiento 0,5\% situación fuertemente relacionada con la crisis económica mundial (...) Sin embargo, para el siguiente año se presentó un crecimiento de $5,47 \%$ gracias a la realización de los Juegos Suramericanos, Feria de Flores, entre otros eventos, (...) [en 2010] se observó una ocupación de 53,0\%, 
lo que supera en 5,2 puntos porcentuales el compendio del año anterior (Anuario Estadístico de Antioquia, 2011b).

Con respecto a los establecimientos comerciales ${ }^{6}$ en Antioquia, en 2004, existían 1.321 y posteriormente para el 2011 ascendieron a 2.052. De estos, en 2013 existían en todo el departamento de Antioquia un total de 864 establecimientos para el alojamiento y hospedaje de turistas, de los cuales 150 (es decir el 17.3\%) estaban ubicados en el Área Metropolitana. Con respecto a los indicadores de conectividad, se tiene que para el 2012, luego de la consolidación de los datos del 2011, el número de hogares con acceso a internet en Antioquia era de 709 mil, de los cuales, 677 mil pertenecían a la zona urbana (Dane, 2012). En el referente nacional, Antioquia también ocupó el segundo lugar, después de Bogotá, en cuanto al porcentaje de hogares con conexión a internet (39\%) y porcentaje de hogares que poseen computador (46\%).

Así, algunos de los retos más importantes para el caso del departamento de Antioquia serían, en primer lugar, la coordinación multinivel, para potenciar a cada uno de los 125 municipios que lo componen, algunos con más potencialidades para el turismo o mayor infraestructura para eventos internacionales. Es decir, el reto está en conocer las capacidades institucionales, de infraestructura, de capital humano y oferta local relacionada con las actividades que se prioricen para la internacionalización. Un segundo reto, tiene que ver con la articulación y corresponsabilidad de Medellín y los municipios que componen el Área Metropolitana $^{7}$ frente a la internacionalización de la región.

Particularmente, en el marco de la Alianza Medellín-Antioquia ${ }^{8}$ para el período 2012-2015, se definió la Cooperación, inversión e internacionalización: las fortalezas de Medellín compartidas con la Región como un área estratégica para la internacionalización de Medellín y la Región, lo que permitiría que se reconozca el proceso que se viene realizando la ciudad en el contexto internacional, sino que también permitirá la inversión de capital extranjero en la economía local.

\footnotetext{
6. Incluye establecimientos de industria, comercio y servicios de las zonas urbana y rural.

7. establecimientos de industria, comercio y servicios de las zonas urbana y rural. Se trata de: Caldas, La Estrella, Itagüí, Sabaneta, Bello, Copacabana, Girardota y Barbosa.

8. La Alianza Medellín-Antioquia o Alianza AMA, se concibió como una apuesta política, entre los gobiernos local y regional, para la atención conjunta de los problemas de Medellín y Antioquia. Dicha alianza se presentó a la sociedad como una alianza histórica, una oportunidad única de corte programático, para afrontar los principales problemas de la ciudad y el departamento conjuntamente, más que una alianza para sumar votos. Según Aníbal Gaviria, la Alianza le permitiría gobernar con participación ciudadana. Mientras que Fajardo asumió la Alianza como un pacto contra la corrupción.
} 
Sin embargo, la coordinación entre los dos niveles de gobierno para esta meta ha pretendido más que una colaboración conjunta para la definición de estrategias de internacionalización, un "derrame” de los beneficios de la internacionalización de Medellín a los municipios antioqueños más cercanos a la capital.

El mayor esfuerzo económico y estratégico lo realiza, entonces, Medellín, aprovechando la institucionalidad que ha sido creada para ese fin; mientras que Antioquia aprovecha parte de esos beneficios, aunque estos se concentren en municipios como Rionegro y otros del oriente cercano, aprovechando su cercanía con el aeropuerto internacional y zonas turísticas como La Ceja y Santa Elena. También se benefician municipios como Santafé de Antioquia, por su tradición turística y el asentamiento en este de casas y hosterías de recreo para personas con alto poder adquisitivo. Finalmente, municipios como Guatapé, también vienen creciendo en la recepción de turistas, debido a su riqueza natural y sitios para la realización de actividades acuáticas.

\section{Conclusiones}

El rol del gobierno departamental de Antioquia durante el período 20042011 se ha centrado en promover la internacionalización de Medellín y la del departamento. Esta actuación es coherente con el modelo de autoridad coordinada propuesto por Wright (1997), y con el propósito de fortalecer el relacionamiento intergubernamental. Este objetivo se ha mantenido en la agenda gubernamental mediante la formulación de planes y programas que relacionan la integración regional y la internacionalización, con miras, principalmente al mejoramiento de la competitividad de la región. Dicha competitividad es necesaria para la atracción de inversión, así como de acuerdos y convenios con organizaciones y empresas exteriores, de tal manera que se incremente el flujo comercial y económico y, por lo tanto, el bienestar de la región. Se trata, pues, de una de las estrategias naturales que utilizan este tipo de gobiernos para su internacionalización, tal como lo expone Ugalde (2006) y que están propuestas desde la lógica de la glocalización, según Soja (2008), en la que busca que Antioquia participe de los flujos de capital global.

Para lograr la materialización de esta meta, la Gobernación de Antioquia ha creado, aunque si bien una incipiente institucionalidad que funciona más desde la voluntad política, unas alianzas estratégicas a través de la Comisión Tripartita, la Alianza de Voluntades y la Alianza Medellín-Antioquia para impulsar el nuevo 
modelo de desarrollo de Antioquia, el cual tiene como propósito insertarse en la economía global mediante el incremento de competitividad.

Particularmente, a estas agencias se les encomienda las funciones y actividades promocionales y de fomento exterior, de manera que la región no se margine del mercado y los flujos de capitales que la globalización moviliza. Así mismo, se les encomienda la función de mejorar el relacionamiento multinivel, que para el caso del objetivo de la internacionalización, los resultados adquiridos por Medellín, le permiten una participación al departamento, mediante actuaciones conjuntas, como sucede con eventos deportivos internacionales. Entonces, así como Farías (2014) habla de un hermanamiento de ciudades, lo que agencias como la Comisión Tripartita buscan en Antioquia, es un hermanamiento entre la ciudad y el departamento.

Se reconocen esfuerzos realizados en el período 2004-2011 a través de la implementación de programas orientados al mejoramiento de la infraestructura vial y de transporte; así como al mejoramiento de sistemas productivos locales y las exportaciones. En el período 2012-2015, con la visión de implementar en Antioquia políticas públicas y programas que fueron exitosos en Medellín, se busca poner a Antioquia en el mapa del mundo, mediante la atracción de inversión y empresas internacionales que se instalen en Antioquia. Para ello, se han buscado relaciones con agencias como la European Commission, el Banco Mundial, el Banco Interamericano de Desarrollo, el Programa de las Naciones Unidas para el Desarrollo, la USAID (United States Agency Internacional Development), entre otras.

La internacionalización de Antioquia se ha hecho por la vía de “derrame” de los beneficios de la internacionalización de Medellín a los municipios antioqueños más cercanos a la capital. Ello, devela las dificultades que existen en materia de coordinación entre los dos niveles de gobierno para la definición de estrategias de internacionalización. Entonces, a pesar de los programas y metas en relación con la integración regional en Antioquia, lograr una internacionalización para el departamento en los términos como se ha logrado para Medellín, no es tarea fácil y requiere de grandes inversiones en infraestructura hotelera y turística, así como el mejoramiento en infraestructura vial y aeroportuaria para las conexiones internas y externas. Además, el reto más importante sigue siendo lograr una real coordinación y articulación multinivel, que permita la corresponsabilidad de Medellín y los municipios que componen el Área Metropolitana para la internacionalización de la región. 


\section{Referencias}

Arandia, I. C. (2002, diciembre). Descentralización y relaciones intergubernamentales en Bolivia. En Reflexión Política, Año 4, No 8. Bucaramanga: Universidad Autónoma de Bucaramanga UNAB. Páginas 7-24.

Borja, J. y Castells, M. (1997). Local y Global: la gestión de las ciudades en la era de la información. Madrid: Editorial Taurus.

Cámara de Comercio de Medellín para Antioquia (s.f.). Estrategia para la internacionalización de Medellín, Área Metropolitana del Valle de Aburrá y Antioquia. Medellín: Cámara de Comercio de Medellín para Antioquia. Tomado de: http://www.camaramedellin.com.co/site/Biblioteca-virtual/ Estudios-economicos/Desarrollo-y-competitividad-regional/Estrategiapara-la-internacionalizacion.aspx. Consultado en mayo 21 de 2015.

Congreso de la República de Colombia (1991). Constitución Política. Bogotá: Gaceta Constitucional No. 116 de 20 de julio de 1991.

Dane-Departamento Nacional de Estadística (2012). Encuesta sobre Tecnologías de la Información y la Comunicación (TIC). Consultado en: http://www. dane.gov.co/index.php/tecnologia-e-innovacion/tecnologias-de-lainformacion-y-las-comunicaciones-tic.

Departamento Administrativo de Planeación (2015). Informe de Rendición de cuentas a junio de 2015. Medellín: Gobernación de Antioquia.

Departamento Administrativo de Planeación (2014). Informe de Rendición de cuentas, 2012-2014. Medellín: Gobernación de Antioquia.

Departamento Administrativo de Planeación (2012).Plan de Desarrollo 20122015: “Antioquia la más educada”. Medellín: Gobernación de Antioquia.

Departamento Administrativo de Planeación (2008).Plan de Desarrollo 20082011: "Antioquia para todos, manos a la obra”. Medellín: Gobernación de Antioquia.

Departamento Administrativo de Planeación (2004).Plan de Desarrollo 20042007: “Antioquia nueva, un hogar para la vida”. Medellín: Gobernación de Antioquia. 
El Colombiano (2013, mayo 8). Medellín se consolida como destino de eventos internacionales. Por: Liliana Vélez, Tomado de: http://www. elcolombiano.com/medellin_se_consolida_como_destino_de_eventos_ internacionales-JBEC_241239.

Farías Peña, J. P. (2014). La paradiplomacia y la construcción de sus bases teóricas. CONfines de Relaciones Internacionales y Ciencia Política, 10 (19), (Enero-Mayo). México: Instituto Tecnológico y de Estudios Superiores de Monterrey. Disponible en:http://www.redalyc.org/articulo. oa?id=63332506009. [Consulta: 13 de marzo de 2015].

García, M. J.; Montilla Martos, J. A. y Arbós Marín, X. (2006). Las relaciones intergubernamentales en el estado autonómico. Madrid: Centro de Estudios Políticos y Constitucionales.

Gobernación de Antioquia (2013b). Anuario Estadístico de Antioquia. Tomado de: http://antioquia.gov.co/images/pdf/anuario_2013/es-CO/capitulos/ poblacion/antioquia/cp-3-2-1.html.

Gobernación de Antioquia (2013a). Anuario Estadístico de Antioquia. Tomado de: http://antioquia.gov.co/images/pdf/anuario_2013/es-CO/capitulos/ ciencia/departamento/innovacion/cp-17-4-1-4.html.

Gobernación de Antioquia (2011a). Anuario Estadístico de Antioquia. Datos sobre transporte. Tomado de: http://antioquia.gov.co/images/pdf/ Cuentas_economicas/es-CO/cuentas-2005-2011/temas/estructura/pib/ transporte.html.

Gobernación de Antioquia (2011b). Anuario Estadístico de Antioquia. Datos sobre comercio.Tomado de: http://antioquia.gov.co/images/pdf/ Cuentas_economicas/es-CO/cuentas-2005-2011/temas/estructura/pib/ comercio.html.

Jordana, J. (2002a). La influencia de las relaciones intergubernamentales en los procesos de descentralización en América Latina. Washington DC: Instituto Interamericano de Desarrollo Social.

Jordana, J. (2002b). Relaciones intergubernamentales y descentralización en América Latina. Proyecto Conjunto Indes Comunidad Europea. Washington: Banco Interamericano de Desarrollo. 
Medellín Convention\&Visitors Bureau (2014). Boletín Conexión Medellín 2014. Medellín: página web oficial. Tomado de: http://www.medellinconventionbureau.com/content/bolet\%C3\%ADn-conexi\%C3\%B3n-medell\%C3\%ADn-0.

Méndez, J. L. (1997). Estudio introductorio. Primera edición en español del libro "Para entender las relaciones intergubernamentales" de Deil S. Wright. México, D.F., Colegio Nacional de Ciencias Políticas y Administración Pública, A.C., Universidad Autónoma de Colima y Fondo de Cultura Económica.

Milani, C.; Ribeiro, M. C. (2010). Paradiplomacia y proyección internacional de las ciudades brasileñas: la elaboración del concepto de "gestión internacional local”. Geopolítica (s). Revista de estudios sobre espacio y poder, (1), 1, p. 23-40.

Molina, A. et al (2012), en su libro resultado de investigación Administración pública y procesos de internacionalización de ciudades. Medellín 1998-2010, exponen en el capítulo 4 el compendio de la normativa nacional y local para el desarrollo de actividades paradiplomáticas en Medellín, páginas 43-59.

Plan Estratégico de Antioquia-Planea (2006a). Hacia un nuevo Modelo de Desarrollo para Antioquia. Bases para la discusión. Segunda edición. Medellín: Plan Estratégico de Antioquia.

Plan Estratégico de Antioquia-Planea (2006b). El Desarrollo Local y Regional de Antioquia. Propuesta estratégica. Segunda edición. Medellín: Plan Estratégico de Antioquia.

Rodríguez, S. (2004). La paradiplomacia: las relaciones internacionales del gobierno de Chiapas. En Presentada para el XVII Congreso Anual de la Asociación Mexicana de Estudios Internacionales. Tuxtla Gutiérrez, Chiapas.

Romero, M. (2005). Aportes para la construcción de un marco de referencia conceptual en el marco de las relaciones Unión Europea-América Latina. En Anuario de la cooperación descentralizada, 2005.Observatorio de la Cooperación Descentralizada Unión Europea-América Latina.

Sassen, S. (2010). Territorio, autoridad y derechos. De los ensamblajes medievales a los ensamblajes globales. Buenos Aires: Katz editores. 
SOJA, E. (2008). Estudios críticos sobre las ciudades y las regiones. Recurso electrónico. Edición Traficantes de Sueños. Licencia Creative Commons.

Ugalde Zubiri, A. (2015). Actores gubernamentales no centrales en las relaciones internacionales. Presentación en Seminario (mayo 13 de 2015), Universidad Nacional de Colombia-Sede Medellín.

Ugalde, A. (2006). La acción exterior de los Actores Gubernamentales No Centrales: un fenómeno creciente y de alcance mundial. Politika Revista de Ciencias Sociales, diciembre de 2006.

Vieira, J. G. (2011). La política de internacionalización de Medellín. Medellín: artículo publicado en el blog del Grupo de Estudios en Ciencia Política y Administración Pública de la Universidad Nacional de Colombia, sede Medellín: http://gecipap.blogspot.com/2011/04/la-politica-deinternacionalizacion-de.html.

Wright, D. (1997). Para entender las relaciones intergubernamentales. México, D.F., Colegio Nacional de Ciencias Políticas y Administración Pública, A.C., Universidad Autónoma de Colima y Fondo de Cultura Económica.

Zapata, O. L. y Vásquez, A. V. (2012, diciembre). Gobernanza en las instancias de interacción y articulación entre el municipio de Medellín y el departamento de Antioquia, 2004-2011. Estudios de Derecho, 154. Facultad de Derecho y Ciencias Políticas, Universidad de Antioquia, (pp. 513-538). 